\title{
Causas de descarte en tres establos lecheros del Valle de Huaura, 2005 - 2010
}

Causes of disposal in three dairy stables of the Huaura Valley, 2005 - 2010

$$
{ }^{1} \text { Carlomagno Velázquez Vergara }{ }^{\mathrm{a}}
$$

\section{RESUMEN}

La presente investigación se realizó con el objetivo de conocer las causas de descarte de vacas lecheras en el valle de Huaura. Se realizó entre enero a diciembre del 2010 y comprendió la evaluación de 2263 registros individuales de tres establos lecheros de crianza intensiva desde el 2005 al 2010. Las causas de descarte se agruparon en seis grupos: problemas reproductivos, ubre, aparato locomotor, peripartales, emergencias y otros. Los datos se analizaron utilizando las medidas de posición central. La principal causa de descarte fue los problemas reproductivos (37.3\%), seguido de problemas de ubre (21.5\%), aparato locomotor $(9.7 \%)$, peripartales $(8.1 \%)$, emergencias $(5.6 \%)$ y otros $(17.9 \%)$. Se concluye que los problemas reproductivos son la principal causa de descarte, seguido de los problemas de ubre y del aparato reproductor.

Palabras clave: vacas, descarte, establos, problemas reproductivos, ubre.

\begin{abstract}
This research was conducted with the objective of knowing the causes of culling of dairy cows in the valley of Huaura. It Was carried out from January to December 2010 and included the evaluation of 2263 individuales records of three intensive-breeding dairy farms from 2005 to 2010. The causes of discard were grouped into six groups: reproductive problems, udder, locomotor system, peripartal, emergencies and others. Data were analyzed using the central position measures. The main cause of discarding was reproductive problems $(37.3 \%)$, followed by udder problems $(21.5 \%)$, locomotor system $(9.7 \%)$, peripartal (8.1\%), emergencies (5.6\%) and others (17.9\%). It Is concluded that reproductive problems are the main cause of discard, followed by udder problems and the reproductive system.
\end{abstract}

keyword:Cows, discarding, stables, reproductive problems, udder

${ }^{1}$ Universidad Nacional José Faustino Sánchez Carrión

${ }^{a}$ Médico Veterinario 


\section{INTRODUCCIÓN}

El valle de Huaura es el principal centro productor de leche en la región Lima provincias. Cuenta con 8000 vacas que producen alrededor de 200,000 litros de leche diarios (MINAG, 2012).

En la actualidad, los cambios en la alimentación y el manejo para obtener una mayor productividad han originado el incremento de algunos problemas sanitarios, que influyen en el descarte de las vacas y son necesarios evaluar para tener un conocimiento real del problema, que luego ayude al ganadero a tomar las mejores decisiones.

Las causas que originan el descarte de vacas han sido estudiadas por Pando, (1969) en la cuenca de Cajamarca; Dextre, (1961), Castagnino, (1983) y Orrego (2001) en la cuenca de Lima, quienes determinaron como principales causas de descarte los problemas reproductivos y baja producción láctea.

El incremento creciente de los costos de producción obliga a los productores lecheros a optimizar el proceso productivo. El descarte de vacas que aun no han completado su ciclo productivo genera pérdidas económicas que es necesario reducirlo implementando medidas sanitarias y de manejo apropiadas. Las vacas deben permanecer en el establo mínimo hasta la tercera lactación, donde expresan el $100 \%$ de su potencial productivo, en las campañas siguientes, la producción de leche disminuye gradualmente. Además, a medida que la vaca envejece, el sistema inmunológico pierde eficiencia predisponiendo a los animales al padecimiento de enfermedades, principalmente Mastitis y Metritis (Philpot and Nickerson, 2000).

El reemplazo de las vacas descartadas tiene costos elevados. En establos lecheros de mayor productividad el valor de una vaquillona oscila entre \$2000 a 3000 dólares. Este sería el costo que el productor tendrá que asumir como reposición por cada vaca que descarta, para mantener una población estable.

La presente investigación tiene como objetivo conocer las causas de descarte en los principales establos lecheros del valle de Huaura, durante el periodo 2005 2010 .

\section{MATERIALES Y MÉTODOS}

La investigación se realizó en tres establos lecheros de crianza intensiva de ganado bovino raza Holstein Friesan; ubicados en el valle de Huaura; provincia de Huaura: región, Lima provincias y comprendió el periodo de enero a Diciembre del 2010. Se evaluaron 2263 registros individuales de vacas descartadas desde el 2005 hasta el 2010. Las causas de eliminación fueron agrupadas teniendo como referencia el trabajo de Milián (1991), donde se considera como vacas descartas a aquellas que salieron en pie del establo, dejando de 
lado las vacas postradas o muertas en el establo y a aquellas que no tenían registrados correctamente las causas de su eliminación. Las causas de descarte fueron agrupados en seis categorías: Problemas reproductivos: abortos, infecciones del aparato reproductor, fetos momificados, infertilidad, esterilidad. Problemas de ubre: infecciones de ubre, infecciones sistémicas con mastitis por coliformes o estafilococos de curso hiperagudo, cuartos perdidos, ubre con ligamentos vencidos.

Problemas del aparato locomotor o traumatismos: Pérdida de la Integridad Anátomo - Fisiológica (PIAF); cojeras, infecciones de la pezuña, problemas en la cadera.

Problemas peripartales: Trastornos metabólicos (hipocalcemia, acetonemia), partos distócicos y complicaciones secundarias, desplazamiento de abomaso, Problemas de emergencia con riesgo de muerte: Leucosis enzootica bovina, traumatismos de peritoneo y corazón, pericarditis, neumonía fulminante, toxemias, peritonitis aguda.

Otros: Reactores positivos a pruebas serológicas, tuberculosis, timpanismos, insuficiencia cardiaca congestiva, intoxicaciones, enfermedades de origen hereditario (síndrome espástico). Además, vacas vendidas para recría, intervenidas por cirugía, o con una baja producción láctea y edad avanzada.

Los datos recolectados se vaciaron en una hoja EXCEL y se analizaron con el programa MINITAB v.16. Mediante las medidas de posición central: promedios y porcentajes; se presentan las causas de descarte de vacas agrupadas en seis categorías.

\section{RESULTADOS YDISCUSIÓN}

Los resultados se detallan en la tabla 1 . Las tres principales causas de descarte fueron: Los trastornos reproductivos, $37.3 \%$, la primera causa de descarte en vacas; seguido de problemas de la ubre, $21.5 \%$ y trastornos del aparato locomotor. En relación a las evaluaciones realizadas en años anteriores, se registra un incremento del descarte por problemas de ubre y de locomoción mientras que el descarte por baja producción y edad avanzada han disminuido.

Los resultados obtenidos confirman los obtenidos por Pando, (1969); Dextre, (1961); Castagnino, (1983) y Orrego (2001), quienes reportaron los problemas reproductivos como la principal causa de eliminación de vacas en los establos.

En relación a los trastornos reproductivos, las principales causas de descarte fueron la infertilidad (58.1\%) y el aborto (22.5\%). La infertilidad representa el descarte de vacas repetidoras que luego de varias inseminaciones no lograron preñar ó se produjo la muerte embrionaria temprana. La menor fertilidad de las vacas está asociada con el aumento de la productividad. A medida que incrementa la producción de leche disminuye la fertilidad de las vacas. 
Esta relación inversa, es consecuencia del balance negativo de energía que sufre la vaca durante los primeros 60 días postparto que originan trastornos metabólicos (Hansen, 2000) y alteraciones hormonales que generan el funcionamiento anormal de los ovarios (Wiltbank et al, 2006); además, la disminución de la fertilidad está asociada con el incremento de la consanguinidad en el ganado de raza Holstein (Hansen, 2000),

La tasa de vacas descartadas por aborto es elevada, muy por encima del 8\%, considerada como normal (Andressen, 1999), puede estar relacionada con algunos patógenos como la Diarrea viral bovina y Neosporosis, enfermedades diagnosticadas en el valle de Huaura, y que son los principales agentes infecciosos responsables de la presentación del aborto bovino en la cuenca de Lima (Rivera, et al., 2001).

Los problemas de ubre (21.5\%) constituyen la segunda causa de descarte, los resultados obtenidos fueron mayores a los reportados por Orrego et al (2001), quienes en un estudio similar concluyeron que los problemas de ubre (9.4\%) constituyen la segunda causa de descarte. Los resultados además, no son coincidentes con los encontrados por Pando, (1969), Dextre, (1961) y Castagnino, (1983), quienes reportan la baja producción láctea como la segunda causa de descarte.

Dentro de los problemas de ubre, la mastitis crónica y la ubre pendulosa constituyen las principales causas de descarte. En general, la mastitis crónica es consecuencia de Mastitis agudas que no respondieron a los medicamentos (Philpot and Nickerson, 2002) y tiene relación con la elevada prevalencia de Mastitis subclínica, que en el valle de Huaura es de 47\% (Velásquez y Vega, 2011). La ubre pendulosa es ocasionada por la rotura del ligamento suspensor, la ubre se descuelga, haciéndola más propensa a sufrir traumatismos y Mastitis; por esta razón, los ganaderos lo descartan de inmediato. Según, Blowey and Edmondson (1995), este problema es de carácter hereditario y la única manera de corregirlo es utilizando toros que mejoren esta característica.

Con relación a los problemas del aparato locomotor $(9.7 \%)$, su presentación es mayor al 3\% reportado por Orrego et al (2001) y está asociado a problemas de manejo. La PIAF (fracturas, luxaciones, claudicaciones, desgarros) fueron la principal causa de descarte, $62.2 \%$; seguido de descaredamiento, $11,6 \% \mathrm{y}$; ataxia de miembros posteriores, $4.6 \%$.

Los problemas peripartales $(8.1 \%)$, que originaron el mayor descarte de vacas fueron el desplazamiento de abomaso y los trastornos metabólicos. Estos trastornos son consecuencia del uso de elevadas cantidades de concentrado en la alimentación con la finalidad de obtener mayor producción de leche (Risco and Archibald, 2010). 
En la categoría de emergencias (5.6\%), se encuentran los trastornos que obligan a un descarte prematuro afectando la vida productiva de los animales. Leucosis enzootica y Pericarditis traumática fueron los principales problemas. En el caso de la leucosis su mayor presentación está relacionado con las altas prevalencias de esta enfermedad en los establos (Andressen, 1999). La presentación de pericarditis traumática está relacionada a deficiencias en la limpieza de los comederos, donde clavos y/o alambres pueden estar mezclados con los forrajes y ser ingeridos por la vaca (Merck, 2004).

En la categoría otros (17.9\%) se agrupan a las causas de descarte con menor presentación. La eliminación de vacas viejas $(6 \%)$ y por baja producción láctea (4.4\%) son las más importantes en este grupo. En condiciones de manejo y sanidad adecuados estas dos causas deberían ser las principales causas de descarte de vacas en los establos lecheros

Se concluye que las causas principales de descarte de vacas lecheras en tres establos del valle de Huaura son los problemas reproductivos, seguido por los problemas de ubre y aparato locomotor.

\section{REFERENCIAS BIBLIOGRÁFICAS}

Andressen, H. (1999). Neosporosis en el Perú y el mundo. Revista de Ciencias Veterinarias. Vol. 15(4),
Lima.

Blowey R. and Edmondson P. (1995). Control de la Mastitis en granjas de vacunos de leche. $1^{a}$ ed. Zaragoza: Acribia. 208 p.

Castagnino, O. (1983). Algunos factores que influyen sobre la eficiencia reproductiva de vacas en establos lecheros de la asociación de Criadores Holstein registrado del Perú, 1981-1982. Tesis de Bachillerato, Facultad de Medicina Veterinaria, Universidad Nacional Mayor de San Marcos, Lima, 39p.

Dextre, E. (1961). Edad al primer parto, longevidad y motivos de eliminación de las vacas lecheras en el valle de Lima. Tesis bachillerato. Facultad de Medicina Veterinaria, Universidad Nacional Mayor de San Marcos, Lima 39p.

Milián, F.(1991). Principales razones de desecho en un hato lechero de 100 vacas. Revista Veterinaria México. 22(2):169-175.,

MINISTERIO DE AGRICULTURA. (2012). Dirección General de Estadística e Informática. En www.minag.gob.pe/download/pdf/e speciales/dinamica/IV_Pecuario.pd f.

Orrego, A. (2001). Principales causas de eliminación de vacas Holstein en Lima. Rev Inv Vet Perú, suplemento 1:329-331.

Pando, L. (1969). Edad al primer parto, longevidad y motivos de eliminación de las vacas lecheras en 
en la campiña de Cajamarca. Tesis bachillerato. Facultad de Medicina Veterinaria, Universidad Nacional Mayor de San marcos, Lima 39p. Philpot, W. and S. Nickerson. (2002). Ganando la lucha contra la Mastitis. Publicado por Wesfalia Surge, Inc. y Wesfalia Landtechmick GmbH.

Risco CA, Archibald LF. (2010). Eficiencia reproductiva en el ganado lechero University of Florida, EE.UU. 5p. Disponible en: htt p: / / www.produccion animal.com.ar

Rivera H. (2001). Etiología infecciosa del aborto bovino. Revista de Investigaciones Veterinarias del Perú, suplemento 1.

Velásquez C. y Vega J. (2012). Calidad de leche y Mastitis subclínica en establos de la provincia de Huaura, Lima. Rev Inv Vet Perú: 23(1):6571.

Wiltbank MC, Lopez H, Sartori R, Sangsritavong S, Gümen A. (2006). Changes in reproductive physiology of lactating dairy cows due to elevated steroid metabolism. Theriogenology; 65 : 17-29......(2004). Manual Merck de Veterinaria. Edit. OceanumCentrum, $5^{\text {ta }}$ edición. 


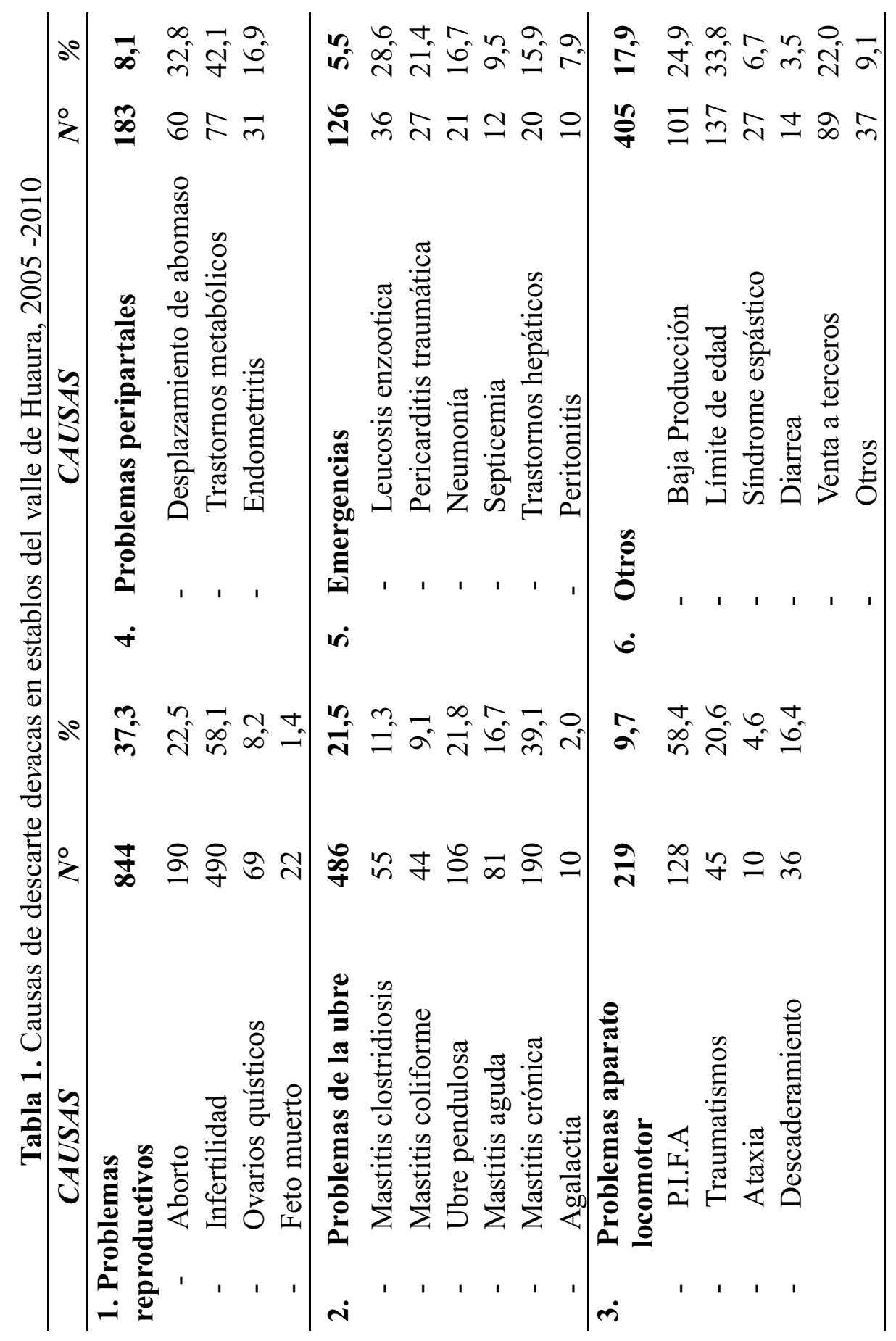

CORRESPONDENCIA:

Dr. Carlomagno Velázquez Vergara cvvergara11@hotmail.com 